\title{
On gauge invariant nucleon spin decomposition
}

\author{
P.M. Zhang ${ }^{1,2}$ and D.G. Pak ${ }^{1,3, a}$ \\ 1 Institute of Modern Physics, Chinese Academy of Sciences, Lanzhou 730000, China \\ 2 Research Center for Hadron and CSR Physics, Lanzhou University and Institute of Modern Physics of CAS, Lanzhou 730000, \\ China \\ 3 Bogoliubov Laboratory of Theoretical Physics, Joint Institute for Nuclear Research, Dubna, Moscow region, 141980, Russia
}

Received: 6 March 2012 / Revised: 28 May 2012

Published online: 25 June 2012

(c) The Author(s) 2012. This article is published with open access at Springerlink.com

Communicated by M. Anselmino

\begin{abstract}
A non-uniqueness problem of gauge invariant separation of quark and gluon contributions to nucleon spin is considered. We show that there is a wide number of gauge invariant spin decompositions, and each of them reduces to the canonical one in a special gauge. A class of physical gauge equivalent nucleon spin decompositions is selected by requirements of consistence with helicity notion described within the $E(2)$ little group representation theory and with the gluon helicity $\Delta g$ measured in the experiment.
\end{abstract}

It has been a long-standing problem of gauge invariant definition of gluon spin and orbital angular momentum [1, $2]$. Recently a gauge invariant decomposition of the total nucleon angular momentum into quark and gluon constituents has been proposed [3,4], and subsequently other possible gauge invariant decompositions for nucleon spin have been suggested [5-9]. Despite this progress there are still principal controversies on the fundamental conceptual level in determining a consistent notion for gluon spin and orbital angular momentum [10,11]. In this letter we revise the problem of nucleon spin decomposition and the existence of a consistent gauge invariant concept of spin in the non-Abelian gauge theory.

Let us start with the well-known canonical decomposition of the nucleon total angular momentum in quantum chromodynamics (QCD),

$$
\begin{aligned}
J_{\mu \nu}^{c a n}= & \int \bar{\psi} \gamma^{0} \frac{\Sigma_{\mu \nu}}{2} \psi \mathrm{d}^{3} x-i \int \bar{\psi} \gamma^{0} x_{[\mu} \partial_{\nu]} \psi \mathrm{d}^{3} x \\
& -\int \vec{A}_{[\mu} \cdot \vec{F}_{\nu] 0} \mathrm{~d}^{3} x-\int \vec{F}_{0 \alpha} \cdot x_{[\mu} \partial_{\nu]} \vec{A}_{\alpha} \mathrm{d}^{3} x
\end{aligned}
$$

where we use vector notations for vectors in color space. All terms in this decomposition, except the first one, are not gauge invariant. In a series of papers $[3,4,12]$ Chen et al. have proposed a gauge invariant decomposition of the total angular momentum in quantum electrodynamics (QED) and QCD. The basic idea in the Chen et al. approach is to separate pure gauge and physical degrees of freedom of the gauge potential in a gauge covariant way.

\footnotetext{
a e-mail: dmipak@gmail.com
}

Let us rewrite the canonical angular momentum using a more general split of the gauge potential into two independent parts,

$$
\vec{A}_{\mu}=\vec{B}_{\mu}+\vec{Q}_{\mu}
$$

where the field $\vec{B}_{\mu}$ is a so-called background (classical) field, and $\vec{Q}_{\mu}$ is an analog of the quantum field in the framework of the covariant background quantization formalism $[13,14]$. Notice, the background field $\vec{B}_{\mu}$ transforms as a gauge potential whereas the quantum field $\vec{Q}_{\mu}$ transforms as a covariant color vector under the classical type of gauge transformation $[13,14]$. Such a general form of splitting is useful in constructing nucleon spin decomposition schemes with the dynamic quark momentum [7, $8,15]$. Adding a surface term,

$$
\int \mathrm{d}^{3} x \partial_{\alpha}\left(\vec{F}_{0 \alpha} \cdot x_{[\mu} \vec{B}_{\nu]}\right)
$$

to the canonical angular momentum (1), and using the equation of motion

$$
D^{\mu} \vec{F}_{0 \mu}=i \bar{\psi} \gamma^{0} \psi
$$

one can obtain the following expression for the total angular momentum tensor:

$$
\begin{aligned}
J_{\mu \nu}^{c a n}= & \int \mathrm{d}^{3} x\left\{\bar{\psi} \gamma^{0} \frac{\Sigma_{\mu \nu}}{2} \psi-i \bar{\psi} \gamma^{0} x_{[\mu} \mathcal{D}_{\nu]} \psi-\vec{F}_{0[\mu} \cdot \vec{Q}_{\nu]}\right. \\
& \left.-\vec{F}_{0 \alpha} \cdot x_{[\mu}\left(\mathcal{D}_{\nu]} \vec{Q}_{\alpha}-\overrightarrow{\mathcal{F}}_{\nu] \alpha}(B)\right)\right\}
\end{aligned}
$$


where $\mathcal{D}_{\mu}$ contains the background field $\vec{B}_{\mu}$, and $\overrightarrow{\mathcal{F}}_{\nu \alpha}(B)$ is a field strength defined in terms of $\vec{B}_{\mu}$ only. The given expression for the total angular momentum is quite general, and it is valid irrespectively of further imposing any constraints on the fields $\vec{B}_{\mu}, \vec{Q}_{\mu}$. In particular, one can identify the field $\vec{B}_{\mu}$ with a pure gauge field $\vec{A}_{\mu}^{\text {pure }}$ and the field $\vec{Q}_{\mu}$ with a physical gauge potential $\vec{A}_{\mu}^{p h y s}$ by imposing two conditions,

$$
\begin{aligned}
\overrightarrow{\mathcal{F}}_{\mu \nu}\left(A^{\text {pure }}\right) & =0, \\
\mathcal{D}_{i} \vec{A}_{i}^{\text {phys }} & =0,
\end{aligned}
$$

where Latin letters are used for space-like indices. With this, the spin decomposition (5) reproduces the gauge invariant decomposition for the nucleon angular momentum proposed in [4]. It has been shown that the constraints (6) can be solved by perturbation theory producing a solution for $\vec{A}_{\mu}^{p h y s}, \vec{A}_{\mu}^{p u r e}$ in terms of the unconstrained gauge potential [12]. Notice, explicit solutions for $\vec{A}_{\mu}^{p h y s}, \vec{A}_{\mu}^{\text {pure }}$ represent non-local functionals of the initial gauge potential $\vec{A}_{\mu}$. The final expression for the gauge invariant decomposition of nucleon spin corresponds to the space vector part of eq. (5) [4]. Each term in the decomposition has become gauge invariant due to the covariant transformation law for the physical field $\vec{A}^{p h y s}$. In the gauge $\vec{A}^{\text {pure }}=0$ the decomposition reduces to the canonical one in the standard Coulomb gauge. Even though the given decomposition is gauge invariant, it is not satisfactory since the basic constraint (6) defining the notion of the physical gauge potential $\vec{A}_{\mu}^{p h y s}$ is not Lorentz invariant. This might imply that the matrix elements of the spin density operator and orbital angular momentum will be frame dependent. Another serious problem is whether such a decomposition of nucleon spin is unique.

The gauge and Lorentz invariant nucleon spin decomposition has been suggested recently in [7]. The defining equation for the physical field is given by the constraint of Lorenz gauge type

$$
\mathcal{D}^{\mu} \vec{A}_{\mu}^{p h y s}=0 .
$$

There are other known Lorentz invariant gauge conditions, the Gervais-Neveu and Fock-Schwinger gauges. Notice, the Fock-Schwinger gauge $x^{\mu} A_{\mu}=0$ allows to express the vector potential in terms of the field strength in a simple way ${ }^{1}[16,17]$

$$
A_{\mu}=\int_{0}^{1} \mathrm{~d} \alpha \alpha x^{\nu} F_{\nu \mu}(\alpha x) .
$$

Unfortunately, it lacks the invariance under translations. So, the Lorenz-gauge-type constraint is a unique gauge which satisfies the Poincaré and conformal symmetries. A solution to the constraint (7) for $\vec{A}_{\mu}^{p h y s}$ in terms of unconstrained gauge potential can be obtained by the perturbation method in a similar manner as in the case of Chen et

\footnotetext{
${ }^{1}$ Private communication with W.M. Sun, F. Wang suggesting the spin decomposition with the Fock-Schwinger-type con-
} straint. al. decomposition [12]. However, solving the Lorenz gauge constraint (7) on mass-shell, i.e., on the space of solutions to equations of motion, encounters a serious problem. The problem becomes evident in the case of the Maxwell theory where the formal solution is given by

$$
A_{\mu}^{\text {phys }}(\vec{x}, t)=\int \mathrm{d}^{3} \vec{x}^{\prime} \frac{\partial^{\nu} F_{\nu \mu}\left(x^{\prime}, t-\left|\vec{x}-\vec{x}^{\prime}\right| / c\right)}{\left|\vec{x}-\vec{x}^{\prime}\right|} .
$$

In the case of the absence of matter fields the r.h.s. of the equation vanishes identically due to the equations of motion, so that the physical field $A_{\mu}^{p h y s}$ cannot be determined. This is a well-known consequence of the incompleteness of the Lorenz gauge. So, one has to impose an additional condition to provide the transversality property of the real photon (gluon). One may choose, for instance, the Coulomb gauge constraint and solve it as Chen et al. did, but then we shall return to the problem of the Lorentz frame dependence. The problem becomes worse since the choice of the Lorentz non-invariant constraint for $\vec{A}_{\mu}^{p h y s}$ is not unique unlike the case of Lorenz gauge condition.

Each spin decomposition corresponding to a special constraint for physical field defines a class of gauge equivalent operators $\left\{\vec{A}_{\mu}^{p h y s}\right\}$. Since the physical field $\vec{A}_{\mu}^{p h y s}$ is gauge covariant it can be expressed in terms of the field strength and its covariant derivatives. However, it is known that for a given field strength there may exist gauge non-equivalent potentials $[18,19]$. This implies that various $\vec{A}_{\mu}^{p h y s}$ given as solutions to different physical constraints will lead to gauge non-equivalent operators and, in general, to different matrix elements. To select which class of gauge equivalent physical fields $\vec{A}_{\mu}^{p h y s}$ produces a proper spin operator we will require the consistence of definitions for $\vec{A}_{\mu}^{\text {phys }}$ with helicity notion described in the framework of group representation theory. We will demonstrate this by explicitly constructing two different spin decompositions based on using gauge invariant variables and generalized axial-gauge-type constraint for the physical gauge field. Finally we will prove the relation between the gauge invariant definition of gluon spin density and gluon helicity $\Delta g$.

Let us first consider a decomposition scheme based on the known notion of gauge invariant variables in the $S U(2)$ Yang-Mills theory [20]. Main definitions of the gauge invariant variables can be generalized straightforward to the case of $S U(3)$ QCD. The main idea in constructing gauge invariant variables is to find a pure gauge $S U(3)$ matrix field in terms of the initial gauge potential $\vec{A}_{\mu}$. The key observation is that the gauge transformation of the temporal component $A_{0}^{a},(a=1,2, \ldots, 8)$ is given by a covariant time derivative of the gauge parameter. So that $A_{0}^{a}$ can be expressed in a pure gauge form in terms of $S U(3)$ matrix valued function $\mathbf{v}$

$$
\hat{A}_{0}=\mathbf{v}^{-1} \partial_{0} \mathbf{v}
$$

where $\hat{A}_{\mu} \equiv g A_{\mu}^{a} \frac{\lambda^{a}}{2 i}$, and $\lambda^{a}$ are Gell-Mann matrices. Notice, the matrix function $\mathbf{v}$ transforms covariantly under gauge transformation, $\mathbf{g} \in S U(3)$,

$$
\mathbf{v}\left(A^{\mathbf{g}}\right)=\mathbf{v g}^{-1} .
$$


Using eq. (10) and the equation of motion for the temporal component $A_{0}^{a}$,

$$
\begin{aligned}
\left(D_{i}^{2} A_{0}\right)^{a} & =\left(D_{i} \partial_{0} A_{i}\right)^{a}-j_{0}^{a}, \\
j_{0}^{a} & =g \bar{\psi} \gamma^{0} \frac{\lambda^{a}}{2} \psi,
\end{aligned}
$$

one can write down the equation defining the matrix function $\mathbf{v}(A)$

$$
\partial_{0} \mathbf{v}(A)=\mathbf{v}(A)\left(\frac{1}{D_{i}^{2}(A)}\left[D_{j}(A) \partial_{0} \hat{A}_{j}-\hat{j}_{0}\right]\right),
$$

where the equation may include or not the source term.

The solution to eq. (13) can be obtained in the form of the time-ordered exponent [20]

$$
\mathbf{v}(A)=T \exp \left\{\int^{t} \mathrm{~d} t\left(\frac{1}{D_{i}^{2}(A)}\left[D_{j}(A) \partial_{0} \hat{A}_{j}-\hat{j}_{0}\right]\right)\right\} .
$$

This allows to define gauge invariant variables $\hat{A}_{i}^{I}(A)$ and $\psi^{I}(A, \psi)$ in terms of the original gauge potential and matter fields [20]

$$
\begin{aligned}
\hat{A}_{i}^{I}(A) & =\mathbf{v}(A)\left(\partial_{i}+\hat{A}_{i}\right) \mathbf{v}^{-1}(A), \\
\psi^{I}(A, \psi) & =\mathbf{v}(A) \psi .
\end{aligned}
$$

One can check that $\hat{A}_{i}^{I}$ satisfies a constraint which represents a generalized covariant Coulomb gauge condition

$$
D_{i}\left(A^{I}\right) \partial_{0} \hat{A}_{i}^{I}-\hat{j}_{0}=0 .
$$

One should stress that we do not impose this condition, but it is fulfilled identically due to the definitions of $\hat{A}_{i}^{I}$ and $\mathbf{v}(A)$. Since $\hat{A}_{i}^{I}$ and eq. (16) are gauge invariant, the simplest way to prove the identity (16) is to consider the relationship (14) in a special gauge $\mathbf{v}(A)=\mathbf{1}$. Finally, the first equation in (15) can be written in the following inverted form:

$$
\begin{aligned}
\hat{A}_{i} & =\mathbf{v}^{-1}(A) \partial_{i} \mathbf{v}(A)+\mathbf{v}^{-1}(A) \hat{A}_{i}^{I}(A) \mathbf{v}(A) \\
& \equiv \hat{A}_{i}^{\text {pure }}+\hat{A}_{i}^{\text {phys }},
\end{aligned}
$$

where we can identify the first and the second term as pure gauge and physical gauge potentials needed to construct a desired gauge invariant spin decomposition. The temporal components of the pure gauge and physical fields are defined as follows:

$$
\begin{aligned}
& \hat{A}_{0}^{\text {pure }}=\mathbf{v}^{-1}(A) \partial_{0} \mathbf{v}(A), \\
& \hat{A}_{0}^{\text {phys }}=\hat{A}_{0}-\hat{A}_{0}^{\text {pure }} .
\end{aligned}
$$

The corresponding total angular momentum decomposition is given by eq. (5) with the replacement $\vec{B}_{\mu} \leftrightarrow \vec{A}_{\mu}^{\text {pure }}$, $\vec{Q}_{\mu} \leftrightarrow \vec{A}_{\mu}^{p h y s}$. The decomposition reduces to the canonical one in the gauge $\mathbf{v}(A)=\mathbf{1}$, which implies $\vec{A}_{\mu}^{\text {phys }}=\vec{A}_{\mu}$ and the constraint (16) turns into a generalized Coulomb gauge condition for the gauge potential $\vec{A}_{i}$. Quantization with the constraint (16) in the Hamiltonian formalism had been done in [21]. The consistency of the gauges depending on time had been proved in [22]. One should notice that the pure gauge field $\mathbf{v}(A)$ in the absence of dynamic gluons describes also pure gauge Gribov modes which can be separated explicitly [20]. Due to that, there is no Gribov ambiguity problem in the presented decomposition.

The important feature of the spin decomposition based on the gauge invariant variables is that due to eqs. (12) and (13) the time component of the physical field defined in (18) vanishes, $\vec{A}_{0}^{\text {phys }}=0$, before imposing any gauge fixing condition. This allows to prove that the decomposition satisfies the requirement of consistence with the helicity notion from the point of view of the group representation theory. Notice that, due to the condition $\vec{A}_{0}^{\text {phys }}=0$, the constraint (16) in free space can be written explicitly as the transversality condition for the color electric field $\vec{E}_{i}^{I}$,

$$
D_{i}\left(A^{I}\right) \vec{E}_{i}^{I}=0, \quad \vec{E}_{i}^{I}=\partial_{0} \vec{A}_{i}^{I}
$$

The transversality for the physical gauge potential $\overrightarrow{A^{p h y s}}$ will be implied naturally from the helicity gauge conditions considered below. The only frame-independent notion of spin in the gauge theory for a massless particle is the helicity which can be described within the framework of the little group $E(2)$ of the Lorentz group [23]. The gauge invariant consideration of the helicity in QED had been done in [24]. We will generalize the consideration of the helicity notion to the case of non-Abelian gauge theory following the non-covariant treatment of the problem in a similar way as it had been done in the Abelian Maxwell theory [25]. The construction of the physical gauge potential $\overrightarrow{A_{\mu}^{p h y s}}$ plays a crucial role. If the gluon momentum is directed along the $z$-axis, $p_{\mu}=(\omega, 0,0, \omega)$, the generators of the little group $E(2)$ are given by the rotation generator $J_{3}$, which is the helicity operator in this case, and by combinations of boost and rotation generators,

$$
J_{3}, \quad N_{1}=K_{1}-J_{2}, \quad N_{2}=K_{2}+J_{1} .
$$

By definition the transformations of the little group $E(2)$ leave the gluon momentum invariant. For the gauge potential $\vec{A}_{\mu}^{p h y s}$ to represent helicity eigenstates of the operator $J_{3}$ one must have the so-called helicity gauge conditions [25]

$$
\vec{A}_{0}^{\text {phys }}=0, \quad \vec{A}_{3}^{\text {phys }}=0 .
$$

To provide both helicity conditions in a consistent manner with equations of motion has been a principal obstacle toward generalization to the case of the non-Abelian gauge theory. In our approach, since one has the condition $\vec{A}_{0}^{\text {phys }}=0$ on mass-shell by construction, it is possible to provide the second helicity condition $\vec{A}_{3}^{\text {phys }}=0$ by choosing a gauge of either Coulomb or axial or light-cone type. This is the main result which allows to select a physical gauge covariant operator $\vec{A}^{\text {phys }}(A)$ and the corresponding spin density consistently with the helicity notion.

Now, it becomes clear that there should exist a class of gauge equivalent spin decompositions which satisfy on 
mass-shell the same helicity conditions for the physical field. One such possible decomposition has been proposed recently [9]. Let us consider other decomposition schemes with a generalized axial gauge-type constraint for the physical field and without invoking the concept of an invariant field. One can construct such decompositions in a surprisingly simple way. Let us define the physical gauge potential $\vec{A}_{\mu}^{p h y s}$ by a generalized axial gauge-type constraint

$$
n^{\mu} \vec{A}_{\mu}^{p h y s}=0,
$$

where the vector $n^{\mu}$ specifies the axial or light-cone gauge condition. Notice, that the defining eq. (22) for $\vec{A}_{\mu}^{p h y s}$ is similar to a generalized axial gauge condition for the gauge potential used in QCD which admits an explicit solution in terms of the field strength [26]. This allows to write down the expression for the physical gauge field $\vec{A}_{\mu}^{p h y s}$ in terms of the general field strength as follows:

$$
\vec{A}_{\mu}^{p h y s}=-\int_{0}^{\infty} \mathrm{d} \lambda n^{\nu} \vec{F}_{\nu \mu}(x+\lambda n)
$$

A pure gauge field $\vec{A}_{\mu}^{\text {pure }}$ is defined by

$$
\vec{A}_{\mu}^{\text {pure }}=\vec{A}_{\mu}-\vec{A}_{\mu}^{p h y s} .
$$

One can check that $\vec{A}_{\mu}^{\text {pure }}$ satisfies the pure gauge condition $\vec{F}_{\mu \nu}^{\text {pure }}=0$. By choosing the proper vector $n_{\mu}$ one can define the physical gauge potential $\vec{A}_{\mu}^{p h y s}$ by choosing either axial, $\vec{A}_{3}^{p h y s}=0$, or light cone, $\vec{A}_{+}^{p h y s}=0$, equation. Both helicity gauge conditions (21) can be easily reached by imposing the temporal gauge fixing condition $\vec{A}_{0}^{p h y s}=0$. The advantage of the decomposition with the light-cone-type constraint $(22), n^{2}=0$, is that the corresponding non-local operator $\vec{A}_{\mu}^{p h y s}(A)$ reduces to the canonical spin density operator in a special gauge $\vec{A}_{\mu}^{\text {pure }}=0$, i.e., explicitly in the light-cone gauge. This allows to make straightforward one-to-one correspondence of the gauge invariant spin density operator to the gluon helicity $\Delta g$ measured in experiment. Notice, that gauge invariant spin density operator in other known spin decomposition schemes reduces to the canonical one in a different gauge. Let us write the four-vector for gluon spin operator corresponding to the canonical gluon spin density

$$
S_{\mu}^{\text {gluon }}=\epsilon_{\mu \nu \rho \sigma} \vec{F}_{\nu \rho} \cdot \vec{A}_{\sigma}^{p h y s}
$$

Substituting (23) into the last equation one can express the spin vector in light-cone gauge in a similar manner as in $[26]$

$$
\begin{aligned}
S_{\mu}^{\text {gluon }}= & \operatorname{Tr} \int_{0}^{\infty} \mathrm{d} \lambda n^{\nu} F_{\nu \xi}(\lambda n+z) \\
& \cdot P \exp \left(i g \int_{0}^{\lambda} \mathrm{d} u n^{\nu} A_{\nu}(u n+z)\right) \tilde{F}_{\xi \mu}(z) \\
& +n_{\mu}\left(\mathcal{O}\left(A^{3}\right)\right),
\end{aligned}
$$

where $\tilde{F}_{\xi \mu}^{a}$ is the dual field strength, and we have omitted the terms coming from the cubic terms in (25) proportional to $n_{\mu}$, since they will not contribute to the matrix element of $n_{\mu} S_{\mu}$ due to light-cone condition $n^{2}=0$. On the other hand, one has a simplified expression for the gluon helicity at light cone $x^{2}=0[27,28]$

$$
\begin{aligned}
(s x) \Delta g= & \langle N| \int_{0}^{\infty} \mathrm{d} \lambda x^{\mu} F_{\xi \mu}(\lambda x) \\
& \cdot P \exp \left(i g \int_{0}^{\lambda} \mathrm{d} u x^{\nu} A_{\nu}(u x)\right) x^{\nu} \tilde{F}_{\nu \xi}(0)|N\rangle
\end{aligned}
$$

where $s_{\mu}=\bar{u}(p, s) \gamma_{\mu} \gamma_{5} u(p, s)$ is the four-vector of nucleon spin. With (26) one results in the known relationship between $\Delta g$ and the nucleon expectation value of the transverse part of $S_{\mu}$

$$
\left\langle N\left|x^{\mu} S_{\mu}^{\text {gluon }}\right| N\right\rangle=-(s x) \Delta g .
$$

In conclusion, there is a wide number of possible gauge invariant spin decompositions suggested in [3-9] and in the present paper. In general they lead to gauge nonequivalent gluon spin operators. The Poincaré and conformal invariance selects a unique Lorentz invariant decomposition with the Lorentz-type constraint for physical field $[7,8]$. However, since this decomposition is not well defined on mass-shell, its physical meaning is unclear. For most of Lorentz non-invariant decompositions the definition of spin operator is frame dependent. We have shown that there is a class of gauge equivalent spin decompositions, eqs. (17) and (23), leading to gauge invariant gluon spin operators consistent with the helicity notion, so that such definitions of spin operators are frame independent. In general, the gauge invariant spin densities represent spatially and temporally non-local functionals. However, this non-locality has no physical meaning since it can be removed in a special gauge resulting in a standard local expression for the canonical spin decomposition. In the practical use, the decomposition defined by (23) and (24) is more suitable. The corresponding definitions for the spin operator are gauge equivalent and lead to the same matrix elements. Notice, that the notion of such defined gauge invariant spin operator with using a non-local operator function for the physical field $\vec{A}_{\mu}^{p h y s}$ is similar to the notion of the quantum effective action which is gauge invariant but has a different operator form depending on a chosen gauge.

As is known, there are two principal issues in the nucleon spin decomposition problem. The first one is how to separate contributions of nucleon constituents. Another one is related to the problem of observability in experiment. In QED the photon spin and orbital angular momentum are measurable quantities [29-33], and they correspond to the canonical decomposition [34-38]. In QCD, since hadrons represent strongly bound states, other schemes of nucleon spin decomposition with dynamic quark momentum might be more relevant $[5-8,15]$. This problem will be considered in a separate paper [39]. 
One of the authors (DGP) thanks N.I. Kochelev, A.E. Dorokhov, I.V. Anikin, O.V. Teryaev, A.V. Efremov for numerous discussions and hospitality during his staying in BLTP, JINR. We thank Y.M. Cho for critical comments and F. Wang, W.M. Sun for numerous interesting discussions. The work was supported by NSFC Grants (Nos. 11035006 and 11175215), and by CAS (Contract No. 2011T1J31).

Open Access This is an open access article distributed under the terms of the Creative Commons Attribution License (http://creativecommons.org/licenses/by/3.0), which permits unrestricted use, distribution, and reproduction in any medium, provided the original work is properly cited.

\section{References}

1. R.L. Jaffe, A. Manohar, Nucl. Phys. B 337, 509 (1990).

2. X. Ji, Phys. Rev. Lett. 78, 610 (1997).

3. X.S. Chen, X.F. Lu, W.M. Sun, F. Wang, T. Goldman, Phys. Rev. Lett. 100, 232002 (2008).

4. X.S. Chen, W.M. Sun, X.F. Lu, F. Wang, T. Goldman, Phys. Rev. Lett. 103, 062001 (2009).

5. M. Wakamatsu, Phys. Rev. D 81, 114010 (2010).

6. M. Wakamatsu, Phys. Rev. D 83, 014012 (2011).

7. Y.M. Cho, M.L. Ge, P.M. Zhang, arXiv:1010.1080.

8. Y.M. Cho, M.L. Ge, D.G. Pak, P.M. Zhang, arXiv: 1102.1130

9. Y. Hatta, Phys. Rev. D 84, 041701(R) (2011).

10. X. Ji, Phys. Rev. Lett. 104, 039101 (2010).

11. E. Leader, Phys. Rev. D 83, 096012 (2011).

12. X.S. Chen, W.M. Sun, F. Wang, T. Goldman, Phys. Lett. B 700, 21 (2011).

13. B.S. DeWitt, Phys. Rev. 162, 1195 (1967).

14. J. Honerkamp, Nucl. Phys. B 48, 269 (1972).
15. D.G. Pak, P.M. Zhang, Consistent gauge invariant nucleon spin decomposition, in Proceedings of the XIV International Workshop on High Energy Physics "DSPIN-11", Dubna, Russia, Sept. 20-24 (2011), edited by A.V. Efremov, S.V. Goloskokov (Publishing Dept. of JINR, Dubna, 2011) pp. 412, arXiv:1204.5030.

16. A.V. Smilga, Sov. J. Nucl. Phys. 35, 271 (1982).

17. V.A. Novikov, M.A. Shifman, A.I. Vainshtein, V.I. Zakharov, Fortsch. Phys. 32, 585 (1984).

18. T.T. Wu, C.N. Yang, Phys. Rev. D 12, 3843 (1975).

19. C.H. Gu, C.N. Yang, Sci. Sinica 20, 47 (1977).

20. V.N. Pervushin, Nuovo Cimento 8, 1 (1985).

21. J. Friedman, N.J. Papastamatiou, Nucl. Phys. B 219, 125 (1983).

22. B.M. Barbashov, A.M. Chervyakov, V.V. Nesterenko, preprint JINR E2-84-521, Dubna (1984).

23. E.P. Wigner, Ann. Math. 149, 40 (1939).

24. S. Weinberg, Phys. Rev. D 134, B882 (1964).

25. D. Han, Y.S. Kim, D. Son, Phys. Rev. D 31, 328 (1985).

26. I.I. Balitsky, V.M. Braun, Phys. Lett. B 267, 405 (1991).

27. A.V. Manohar, Phys. Rev. Lett. 65, 2511 (1990).

28. R. Jaffe, Phys. Lett. B 365, 359 (1996).

29. R.A. Beth, Phys. Rev. 50, 115 (1936).

30. L. Allen, M.W. Beijersbergen, R.J.C. Spreeuw, J.P. Woerdman, Phys. Rev. A 45, 8185 (1992).

31. J. Leach, M.J. Padgett, S.M. Barnett, S. Franke-Arnold, J. Courtial, Phys. Rev. Lett. 88, 257901 (2002).

32. M.F. Andersen et al., Phys. Rev. Lett. 97, 170406 (2006).

33. L. Marrucci, C. Manzo, D. Paparo, Phys. Rev. Lett. 96, 163905 (2006).

34. D. Lenstra, L. Mandel, Phys. Rev. A 26, 3428 (1982).

35. S.J. van Enk, G. Nienhuis, Europhys. Lett. 25, 497 (1994).

36. S.J. van Enk, G. Nienhuis, Phys. Rev. A 76, 053825 (2007).

37. G.F. Calvo, A. Picon, E. Bagan, Phys. Rev. A 73, 013805 (2006).

38. A. Alexandrescu, D. Cojoc, E. Di Fabrizio, Phys. Rev. Lett. 96, 243001 (2006).

39. P.M. Zhang, D.G. Pak, in preparation (2012). 